\title{
La marca personal como elemento decisorio de un buen maestro*
}

\section{The personal mark as a decisive element of a good teacher}

\section{Marca pessoal como elemento decisivo de um bom professor \\ Camila Cortés-Arévalo \\ Profesional en Marketing y Negocios Internacionales. Escuela Internacional de Administración y Marketing - ElAM, Universidad Sergio Arboleda \\ camila.cortes@correo.usa.edu.co}

\section{Santiago Sánchez-Sáenz}

Profesional en Marketing y Negocios Internacionales. Escuela Internacional de Administración y Marketing - EIAM, Universidad Sergio Arboleda,

santiagoe.sanchez@correo.usa.edu.co

\section{Antonio Alonso-González}

Decano Académico de Marketing y Negocios Internacionales, Escuela Internacional de Administración y Marketing - EIAM, Universidad Sergio Arboleda

antonio.alonso@usa.edu.co

\section{Resumen}

La marca personal es el acto de gestionar cómo quiere uno mismo ser recordado. Para las empresas, es muy importante que sus profesionales sean reconocidos y tengan una excelente marca personal que les permita desarrollarse en el ambiente laboral de manera óptima. Lo mismo pasa con las universidades, estas definen la calidad de los cursos que imparten de acuerdo con la calidad de maestro que lo dicte. Es por esta razón que el desarrollo de una marca personal positiva es tan importante para los profesionales en todos los campos y así mismo es necesario definir qué aspectos forman a un buen maestro para tener una mayor recordación en la población universitaria. El presente trabajo trata de identificar estas cualidades que definen a un buen profesor universitario, mediante un estudio de tipo descriptivo, con un enfoque cuantitativo, realizado a partir de una encuesta aplicada sobre estudiantes de la Universidad Sergio Arboleda, obteniendo resultados representativos sobre las cualidades que debe tener un profesor universitario respecto a tres criterios básicos: personales, formativos y docentes, y resaltando la importancia de la gestión de la página web y las redes sociales de la universidad en la creación de la marca personal de dichos profesionales.

* Cómo citar: Cortés-Arévalo, C., Sánchez-Sáenz, S., Alonso-González, A. (2016). La marca personal como elemento decisorio de un buen maestro. Revista Libre Empresa, 13(2), 73-83 http://dx.doi.org/10.18041/libemp.2016.v13n2.26205 


\section{Palabras clave}

Marca personal, maestro, calidad, desarrollo.

\section{Abstract}

Personal branding is the act of managing how you want to be remembered. For some companies it is really important to achieve for their professionals prestige and image, being this reflected as an excellent personal brand that allows them to develop themselves optimally in the work environment. Same thing happens with universities: they define the quality of their courses by the quality of the person who teaches it. This is why the development of a positive personal brand is important for the professionals in every field and likewise is necessary to define which aspects form a good teacher in order to have more remembrance into the university population. This paper attempts to identify these qualities that define a good university professor, through a descriptive study with a quantitative approach, performed from a survey of students at the University Sergio Arboleda, obtaining representative results on the characteristics that must have a university lecturer based on three basic criteria: personal, educational and teaching, highlighting the importance of managing the website and social networks of universities in creating personal brand of these professionals.

\section{Keywords}

Personal branding, professor, quality, development.

\section{Resumo}

Marca pessoal é o ato de administrar-se como você quer ser lembrado. Para as empresas, é muito importante que seus profissionais são reconhecidos e têm excelente marca pessoal que lhes permite desenvolver no local de trabalho de forma otimizada. Mesmo com universidades, estes definem a qualidade de seus cursos de acordo com a qualidade dos ditames dos professores. É por esta razão que o desenvolvimento de uma marca pessoal positiva é tão importante para os profissionais em todos os campos e por isso é necessário definir quais aspectos são um bom professor para ter maior recuperação na população universitária. Este artigo tenta identificar essas qualidades que definem um bom professor universitário, através de um estudo descritivo, com abordagem quantitativa, realizada a partir de uma pesquisa com estudantes da Universidade Sergio Arboleda, obtenção de resultados representativos sobre as qualidades que você deve ter um professor universitário em três critérios básicos: pessoal, educacionais e professores, e destacando a importância da gestão do sítio e sociais redes de universidades na criação de marca pessoal desses profissionais.

\section{Palavras chave}

Marca pessoal, desenvolvimento da qualidade dos professores.

\section{Introducción}

En el mundo globalizado del presente ha venido disminuyendo la brecha entre la realidad física y la realidad virtual, facilitándose las relaciones interpersonales en la distancia y 
otros fenómenos derivados de los avances tecnológicos y la competitividad, siendo cada día más importante destacarse entre los demás. Tal como afirmaba Peters (1997): "la única manera de lograr diferenciarnos en un mundo cada vez más competitivo es manejando nuestra carrera como las grandes empresas manejan las marcas de sus productos”. Por esta razón nace la marca personal.

Históricamente, el concepto adquirió fuerza con el cambio generacional entre los 80's y los 90's, con la crisis económica y la revolución del internet, que dejo como resultado el nacimiento de los llamados "agentes libres", los cuales eran todo un ejército de profesionales cualificados pero descontentos, que se dieron cuenta que las reglas estaban cambiando y que a partir de ese momento, acelerado por la caída demográfica, serían los empleados los que empezarían a fijar las reglas y no las empresas (Pérez \& Marcos, ¿Quién teme al Personal Branding?, 2007). Diferentes autores empezaron entonces a escribir y a definir el concepto de marca personal, como Alonso (2014), que lo describió como el conjunto de atributos fundamentales de una persona y todo aquello por lo que se le reconoce y diferencia de otras y que se representa por su nombre y por su aspecto.

En la misma línea, Arques (2012) estipula que:

La marca personal es la manera de clarificar y comunicar aquello que nos hace diferentes y especiales -y de emplear esas cualidades para guiar nuestra carrera o tomar nuestras decisiones estratégicas-. Se trata de comprender cuáles son los atributos -fortalezas, habilidades, valores y pasiones- que nos hacen únicos y de emplearlos para diferenciarnos de nuestros competidores y de nuestros iguales.

Por otra parte, la imagen de marca es una realidad subjetiva que reside en el contexto mental de los públicos y que Kotler define como el "conjunto integrado de ideas, creencias e impresiones que un consumidor tiene respecto a una marca” (Baños \& Rodríguez, 2012). Esta puede influir decisivamente en la valoración y el comportamiento de los públicos hacia las marcas (Capriotti, 2007).

Como bien es sabido, cada individuo tiene características únicas y diferentes a los demás, y esto es lo que permite que cada uno deje una huella distinta, conocida como su marca personal, compuesta por cuatro pilares definidos por Pérez (2014) como:

- Propósito: para que la marca tenga alguna posibilidad de perdurar debe tener un objetivo claro.

- Perfil: cada huella estará mejor o peor definida o perfilada. Unas mostrarán lo que transmiten y otras quedarán difusas.

- Permanencia: puede que la marca tenga un propósito claro y que esté muy bien definida pero, si no se deja en el sitio apropiado, se perderá.

- Profundidad: es el nivel de penetración en la mente de la audiencia. Cuantas más veces se muestren las cualidades, y en el mayor número de medios posibles, más profundidad se alcanzará y olvidar la marca costará más. 
De acuerdo con Ramírez (2013):

La finalidad del branding personal es posicionarse en la mente de una persona de la mejor forma, como si no existiera competencia alguna. El espacio en la memoria de alguien no necesariamente será ocupado por un producto, servicio o marca, sino también por una persona mediante su branding personal.

Por esta razón, es de vital importancia para las universidades contar con un amplio portafolio de talento humano con reconocimiento en la comunidad académica que le den soporte a la marca de la universidad apoyándose en sus respectivas marcas personales.

Tal como lo afirma (Bain, 2007):

Los profesores extraordinarios conocen su materia extremadamente bien (...) están al día de los desarrollos intelectuales, científicos o artísticos de importancia en sus campos, (...) han utilizado su conocimiento para desarrollar técnicas que les permitan conocer a fondo principios fundamentales y conceptos organizativos que otros pueden utilizar, (...) y es capaz de producir una influencia duradera e importante en la manera en que la gente piensa, actúa y siente.

\section{Metodología}

La investigación es de tipo descriptivo, con un enfoque cuantitativo. Se llevó a cabo una serie de encuestas virtuales utilizando la plataforma Typeform, comprendida en un espacio de duración por encuesta entre cinco y doce minutos. La muestra estuvo integrada por cien estudiantes de universidad, distribuidos entre cuarenta y dos hombres y cincuenta y ocho mujeres, cuyo rango de edad es de 18 a 25 años. La muestra abarcó únicamente a estudiantes de la Universidad Sergio Arboleda, ya que esta fue el objeto de estudio. Se adjuntó el formulario que se puede observar en el Anexo A.

\section{Resultados}

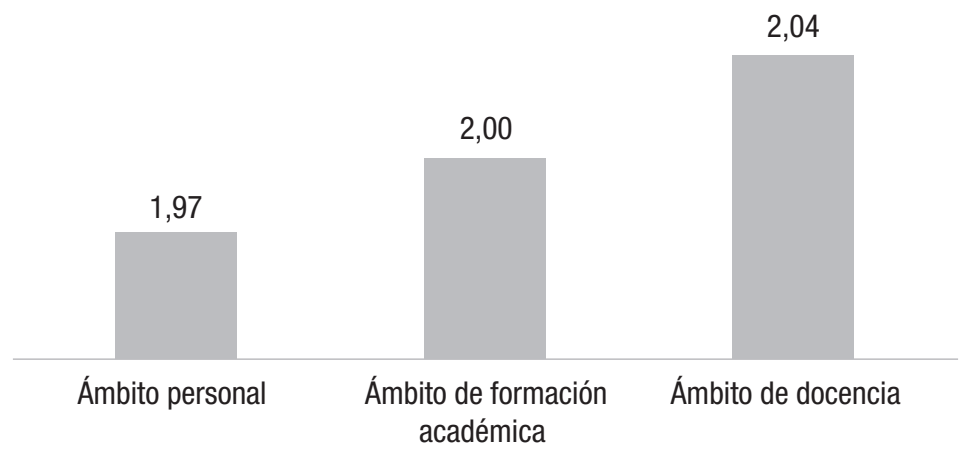

Figura 1. Organice los siguientes ámbitos según la relevancia y efecto de los mismos al momento de elección del profesor 


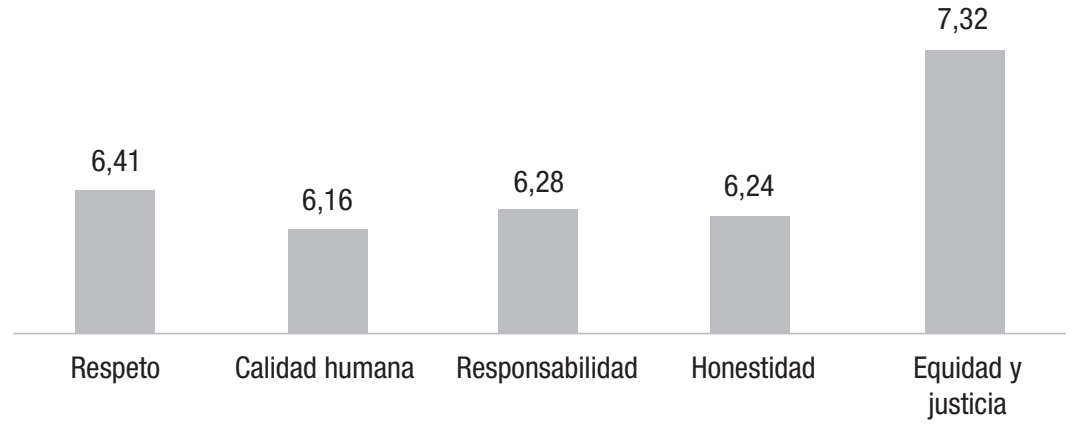

Figura 2. Califique la importancia de las siguientes características en un profesor a la hora de elegirlo, de 1 (mínimo) a 10 (máximo)

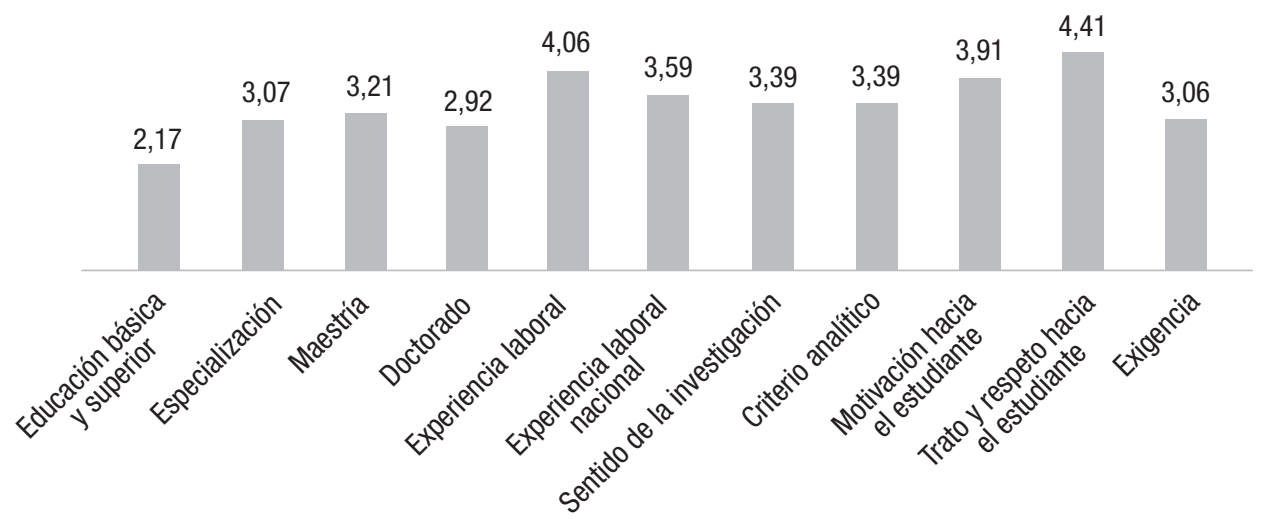

Figura 3. Califique la importancia de las siguientes características en un profesor a la hora de elegirlo, de 1 (mínimo) a 5 (máximo)

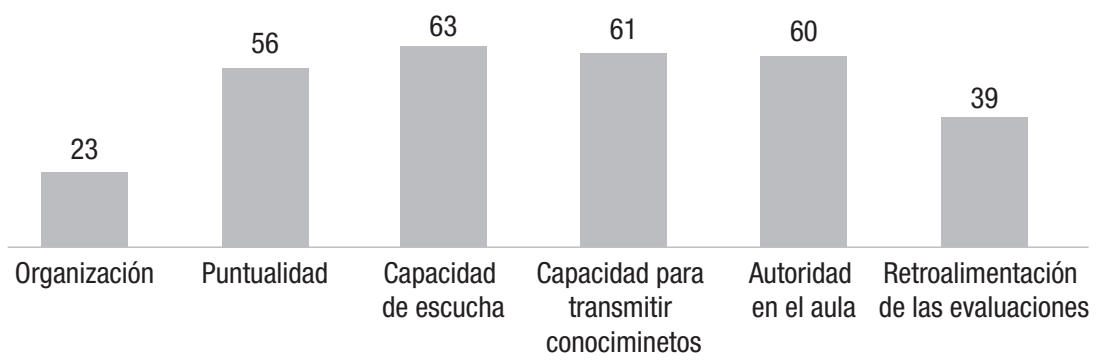

Figura 4. De las siguientes características, escoja las que usted considere son las que mejor describen a un buen profesor 


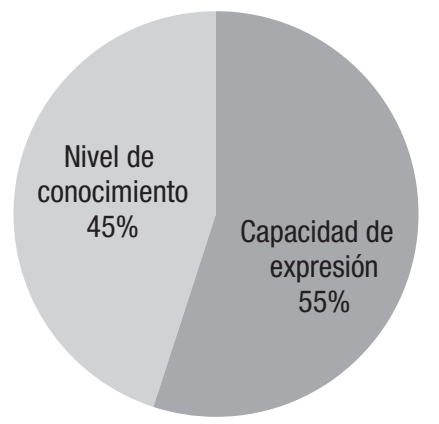

Figura 5. Escoja el factor que para usted tiene mayor relevancia en un buen profesor

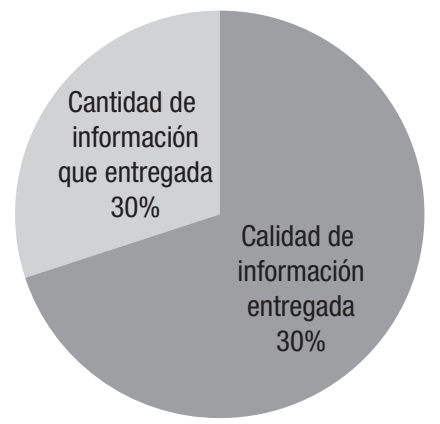

Figura 6. Escoja cuál tiene mayor importancia para usted en un profesor

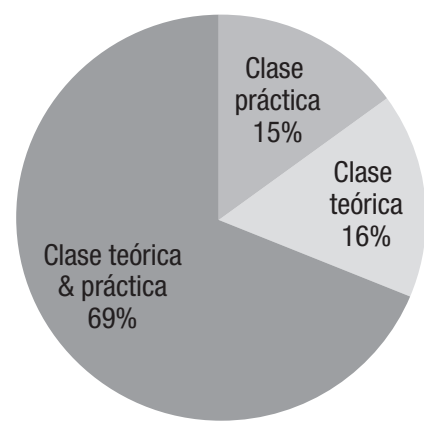

Figura 7. Selecione cuál considera usted que es el mejor método de aprendizaje

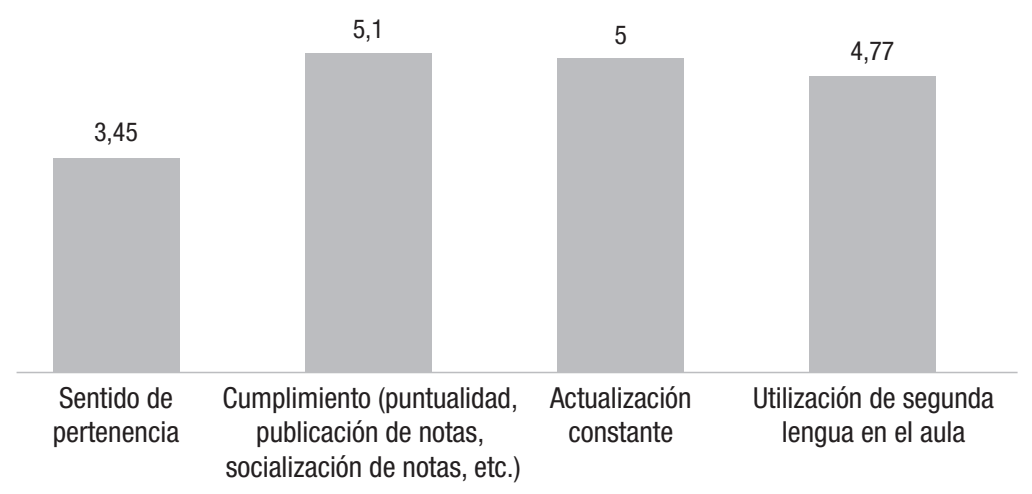

Figura 8. Califique la importancia de las siguientes caraterísticas en un profesor a la hora de elegirlo, de 1 (mínimo) a 7 (máximo)

\section{Librempresa}




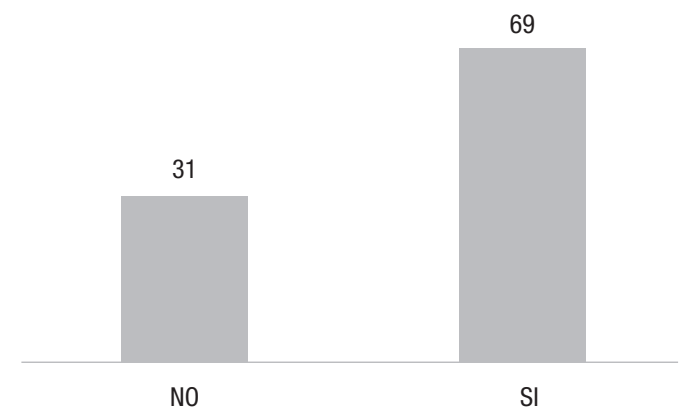

Figura 9. ¿Es importante para usted el prestigio de los profesores al momento de hacer la elección de la universidad?

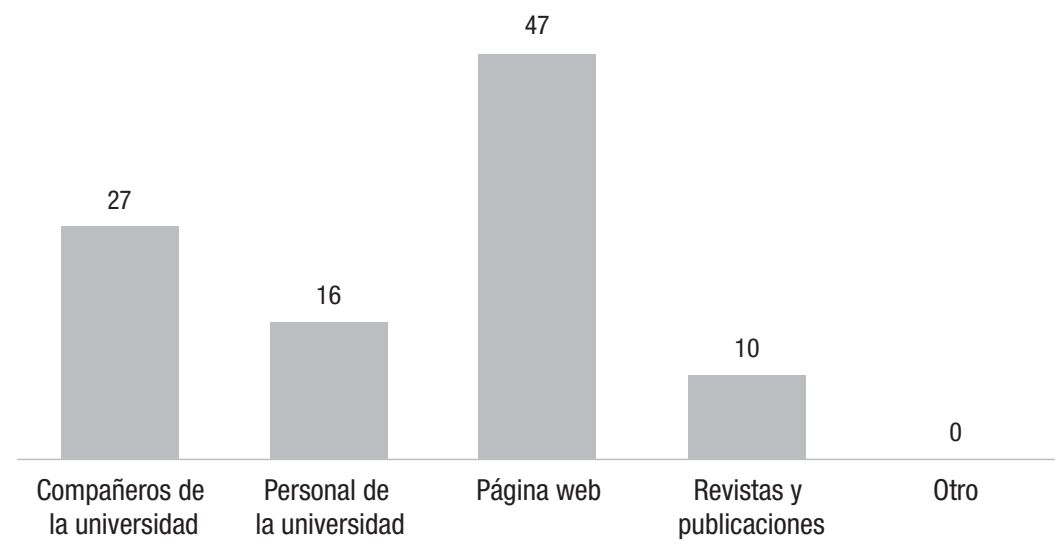

Figura 10. ¿De qué manera se informa usted sobre el prestigio de los profesores?

\section{Análisis de resultados}

Desde el inicio de la investigación se definieron tres pilares sobre los cuales se desarrolló la misma. Después de haber realizado el respectivo análisis de los datos es evidente cómo la teoría mencionada anteriormente es corroborada por los resultados de las encuestas. A continuación se abundará en profundidad sobre estos tres pilares:

1. Criterios personales: Definen la base de toda su formación y profesionalismo. Está ligado a todo lo relacionado con principios, valores, ética y moral.

Como se puede observar en la respuesta de la pregunta número cuatro, los estudiantes encuentran más relevante el trato y respeto que el profesor tiene hacia ellos que la misma educación básica y superior, siendo poco relevante la universidad donde el 
profesor terminó el pregrado. Así mismo, la respuesta de la pregunta tres reafirma que para los estudiantes es importante contar con docentes justos y equitativos, es decir, ceñidos a normas claras y coherentes, guiadas por valores y principios Adicionalmente, los estudiantes encuentran el cumplimiento y la coherencia por parte de los maestros como un factor relevante a la hora de elegirlos ya que se ve relacionado con la transparencia en cada aspecto de su comportamiento.

2. Criterios de formación: La formación da el conocimiento necesario del campo en el que se desenvuelve la persona, son los pilares que dan comienzo a la experiencia adquirida posteriormente. Según la octava pregunta del cuestionario, el 69\% de los estudiantes disfrutan de metodologías dinámicas, las cuales puedan mezclar tanto la parte teórica como la parte práctica de la educación. Adicional a esto, los estudiantes prefieren la calidad sobre la cantidad, es decir, clases que les aporten y les generen impacto y valor agregado. Como aspecto relevante de la categoría, encontramos que la experiencia laboral nacional juega un papel importante en el proceso de elección del maestro por parte del estudiante, ya que esta es una forma aterrizada de ver cómo los conocimientos adquiridos se aplican a casos cercanos y reales.

3. Criterios de docencia: Tener todo el conocimiento sobre el tema no es suficiente para ser un buen profesional, pues es necesario poseer las capacidades para transmitir eficazmente la información. Como se ha observado en toda la investigación, los estudiantes encuentran realmente relevante ser escuchados y tenidos en cuenta, lo que implica el desarrollo de clases más lúdicas. Esto se ve reflejado en la respuesta de la pregunta cinco, en donde los estudiantes escogieron la capacidad de escucha como la principal característica de un buen maestro, seguido por la capacidad de transmitir conocimientos y la autoridad en el aula. Es interesante ver cómo, aunque los estudiantes buscan tener una participación activa en las clases, desean además, contar con un moderador ejemplar que no solo permita la interacción sino que también mantenga el orden.

Según la segunda pregunta del cuestionario, este criterio es el más relevante para los estudiantes. Se puede observar, a través de los resultados obtenidos, que la habilidad de comunicación, la capacidad de expresión, el sentido de pertenencia, entre otros, son aspectos necesarios en un buen maestro.

\section{Conclusiones}

De todo lo expuesto anteriormente podemos concluir que los estudiantes de hoy en día están orientados al orden y la esquematización. Buscan control y manejo oportuno de todas aquellas actividades que les afectan y se relacionan directamente con los maestros (Notas, asistencia, fechas, etc.).

Se pudo observar así mismo que el prestigio de los maestros es básico al momento de los estudiantes seleccionar la universidad donde quieren estudiar, siendo el medio más 
utilizado para informarse sobre el prestigio de los profesores la página web por lo que un excelente manejo de la misma es básico en términos de branding personal y creación de marca de los docentes es fundamental. Hoy en día, el uso de las redes sociales permite no sólo interactuar con distintas personas, sino cumplir la función de una carta de presentación más personalizada, y es por esto que su correcto manejo junto con los contenidos creados en la página web son realmente importantes si se quiere proyectar una buena marca personal.

Por todo ello se considera muy pertinente que las universidades tengan actualizado su portafolio de talento humano, para suplir las necesidades que los estudiantes tienen, siendo adicionalmente necesaria la capacitación del staff frente a la importancia de proyectar una buena marca personal.

\section{Conflicto de intereses}

Los autores declaran no tener ningún conflicto de intereses.

\section{Referencias bibliográficas}

1. Alonso, A. (2014). Personal branding: la importancia de la marca personal. León: Universidad de León.

2. Arqués, N. (2012). y tú, ¿qué marca eres? Barcelona: Alienta.

3. Bain, K. (2007). Lo que hacen los mejores profesores universitarios. Valencia: PUV.

4. Baños, M., \& Rodríguez, T. (2012). Imagen de marca y product placement. Madrid: ESIC.

5. Capriotti, P. (2007). Gestión de la marca corporativa. Buenos Aires: La Crujía.

6. Kotler, P., Kartajaya, H., \& Setiawam, I. (2000). Marketing 3.0. Madrid: LID.

7. Pérez, A. (2014). Marca personal para dummies. Barcelona: Grupo Planeta.

8. Pérez, A., \& Marcos, T. (2007). ¿Quién teme al Personal Branding? Capital Humano, 3.

9. Peters, T. (1997). The brand called you. Fast Company.

10. Ramírez, K. (2013). Branding Personal: Mercadotécnica para el individuo a partir de técnicas del desarrollo del pensamiento creativo. Investigium Ire Volumen 6, 36.

\section{ANEXO A. Formulario}

1. ¿Cuál es su nombre completo?

2. Organice los siguientes ámbitos, de 1 a 3 , siendo 1 el más importante y 3 el menos importante según usted considere, la relevancia y efecto de los mismos al momento de elección del profesor:

a) Ámbito Personal (Está ligado a todo lo relacionado con principios, valores, ética y moral)

b) Ámbito de Formación Académica (Hace referencia a la educación y la experiencia laboral)

c) Ámbito de Docencia (Se relaciona con las habilidades de comunicación, el sentido de pertenencia, la transparencia, etc.) 
3. Califique de 1 a 10 la importancia de las siguientes características en un profesor a la hora de elegirlo, siendo 1 nada relevante y 10 completamente relevante:
a) Respeto
b) Calidad Humana
c) Responsabilidad
d) Honestidad
e) Equidad y Justicia

4. Califique de 1 a 5 , siendo 1 nada relevante y 5 completamente relevante, la importancia de las siguientes características en un profesor a la hora de elegirlo:
a) Educación básica y superior
b) Especialización
c) Maestría
d) Doctorado
e) Experiencia laboral nacional
f) Experiencia laboral internacional
g) Sentido de la investigación
h) Criterio analítico
i) Motivación hacia el estudiante
j) Trato y respeto hacia el estudiante
k) Exigencia

5. De las siguientes características escoja las que usted considere son las que mejor describen a un buen profesor (elija máximo 3 opciones):
a) Organización
b) Puntualidad
c) Capacidad de escucha
d) Capacidad para transmitir conocimientos
e) Autoridad en el aula
f) Retroalimentación de las evaluaciones

6. Escoja el factor que para usted tiene mayor relevancia en un buen profesor:
a) Nivel de conocimiento
b) Capacidad de expresión

7. Escoja cuál tiene mayor importancia para usted en un profesor:
a) Cantidad de información entregada
b) Calidad de información entregada

8. Seleccione cuál considera usted que es el mejor método de aprendizaje:
a) Clase teórica
b) Clase práctica
c) Clase teórica y práctica 
9. Califique de 1 a 7 , siendo 1 nada relevante y 7 completamente relevante, la importancia de las siguientes características en un profesor a la hora de elegirlo:
a) Sentido de pertenencia
b) Cumplimiento (puntualidad, publicación de notas, socialización de notas, etc)
c) Actualización constante
d) Utilización de una segunda lengua en el aula

10. ¿Es importante para usted el prestigio de los profesores al momento de hacer la elección de la universidad?
a) Sí
b) No

11. ¿De qué manera se informa usted sobre el prestigio de los profesores?
a) Revistas y publicaciones
b) Página web
c) Compañeros de la universidad
d) Personal de la universidad
e) Otro 